\title{
CONVEX FUNCTIONS AND THE ROLLING CIRCLE CRITERION
}

\author{
V. SRINIVAS, O.P. JUNEJA and G.P. KAPOOR \\ Department of Mathematics \\ Indian Institute of Technology \\ Kanpur -208016 \\ India
}

(Received May 31, 1991 and in revised form September 7, 1993)

ABSTRACT. Given $0 \leq R_{1} \leq R_{2} \leq \infty, C V G\left(R_{1}, R_{2}\right)$ denotes the class of normalized convex functions $f$ in the unit disc $U$, for which $\partial f(U)$ satisfies a Blaschke Rolling Circles criterion with radii $R_{1}$ and $R_{2}$. Necessary and sufficient conditions for $R_{1}=R_{2}$, growth and distortion theorems for $\operatorname{CVG}\left(\mathrm{R}_{1}, \mathrm{R}_{2}\right)$, and a rotation theorem for the class of convex functions of bounded type, are found.

KEY WORDS AND PHRASES. Univalent functions, Convex functions, Curvature, Subordination, Distortion theorems, Growth theorems. 1991 AMS SUBJECT CLASSIFICATION CODES. 30C45, $30 \mathrm{C5}$.

1. INTRODUCTION.

Let $\underline{S}$ be the class of functions $f(z)$ which are analytic and univalent in the unit disc $U=\{z:|z|<1\}$ and have the normalization $f(0)=0=f^{\prime}(0)-1$. For $f \in \underline{S}$ and $r \in(0,1)$, the radius of curvature, $\rho(z)$ of the curve $f(|z|=r)$ at the point $f(z)$, is given by [6],

$$
\rho(z)=\frac{\left|z f^{\prime}(z)\right|}{\operatorname{Re}\left(1+\frac{z f^{\prime \prime}(z)}{f^{\prime}(z)}\right)}
$$

where $z=r e^{i \theta}$. Goodman [2] introduced the class $c V\left(R_{1}, R_{2}\right)$ of functions $f(z)$ having $\rho(z)$ restricted as $|z|$ tends to 1 . Thus, let

and

$$
\rho_{\star}(r)=\min _{|z|=r} \rho(z), \quad \rho^{\star}(r)=\max _{|z|=r} \rho(z)
$$

$$
R_{\star}=\lim _{r \rightarrow 1^{-}} \rho_{\star}(r) \quad R^{*}=\lim _{r \rightarrow 1^{-}} \rho^{*}(r)
$$

DEFINITION 1. Let $R_{1}$ and $R_{2}$ be fixed in $[0, \infty)$. A function $f \in \underline{S}$ is said to be in the class $C V\left(R_{1}, R_{2}\right)$ if $R_{1} \leq R_{\star}$ and $R^{\star} \leq R_{2}$ where $R_{\star}$ and $R^{\star}$ are as in (1). For $0<R_{1} \leq R_{2}<\infty$, a function $f \in C V\left(R_{1}, R_{2}\right)$ is called a convex function of bounded type.

A function $f(z)$ is said to be in $\overline{C V}\left(R_{1}, R_{2}\right)$ if, $R_{1}=R_{\star}$ and $R_{2}=R^{\star}$, where $R_{\star}$ and $R^{\star}$ are as in (1).

For functions $f(z)$ in the class $C V\left(R_{1}, R_{2}\right)$, Goodman [2] obtained 
(i) the first approximation for the moduli of the Taylor coefficients, (ii) covering theorem and (iii) bounds for $d$, where $d$ is the distance of $\partial f(U)$ from the origin, in terms of $R_{1}$ and $R_{2}$. Goodman [3], Wirths [8] and Mejia and Minda [4] extended this study by finding certain other interesting properties of functions in the class $C V\left(R_{1}, R_{2}\right)$.

styer and wright [7] introduced the following class of functions based on Blaschke's Rolling Circles Criterion:

DEFINITION 2. Given $0 \leq R_{1} \leq R_{2} \leq \infty$ and $R_{2} \geq 1$, let CVG $\left(R_{1}, R_{2}\right)$ be the class of functions $f(z)$ in $\underline{s}$ with the property that for each $\eta \in \partial f(U)$ there are open discs $D_{1}(\eta)$ and $D_{2}(\eta)$ of radius $R_{1}$ and $R_{2}$, respectively, such that, $\eta \in \partial D_{1}(\eta) \cap \partial D_{2}(\eta)$ and

$$
D_{1}(\eta) \subseteq f(U) \subseteq D_{2}(\eta) \text {. }
$$

If $R_{1}=0$ or $R_{2}=\infty, D_{1}(\eta)$ and $D_{2}(\eta)$ are to be interpreted as the empty set and an open half-plane, respectively.

It follows that [7]

$$
\operatorname{CV}\left(R_{1}, R_{2}\right) \subseteq \operatorname{CVG}\left(R_{1}, R_{2}\right) \subseteq C V
$$

where, $\mathrm{CV}$ is the subclass of functions $f(z)$ in the class $\underline{s}$, for which $f(U)$ is convex.

Mejia and Minda [4] showed that, in fact, $\operatorname{CVG}\left(0, R_{2}\right)=\operatorname{CV}\left(0, R_{2}\right)$. However, for $R_{1}>0$, whether $\operatorname{CVG}\left(R_{1}, R_{2}\right)=C V\left(R_{1}, R_{2}\right)$ still holds, remains an open problem. The difficulty to settle this problem lies in the fact that, for $f \in \operatorname{CVG}\left(R_{1}, R_{2}\right), R_{1}>0$, the radius of curvature $\rho(z)$ of the curve $f(|z|=r)$ at the point $f(z)$ may not be a continuous function on $\bar{U}=|z:| z \mid \leq 1$, , (see [7]).

Let $g(z)$ be analytic and univalent in $U$. A function $f(z)$ analytic in $U$, is said to be subordinate to $g(z)$ in $U(f(z) \prec g(z))$ if $f(0)=g(0)$ and $f(U) \subseteq g(U)$.

For a function $f(z)$ in $\underline{s}$, the unit exterior normal to the curve $f(|z|=r)$ at the point $f(z)$ is $n(z)=z f^{\prime}(z) /\left|z f^{\prime}(z)\right|$, where $r \in(0,1)$. Styer and wright [7] found that a normalized univalent function $f \in C V G\left(R_{1}, R_{2}\right)$, if and only if, $f \in C V$, and for every $\zeta \in \partial U$ for which $f(\zeta)$ is finite,

$$
f(U) \leq D\left(f(\zeta)-R_{2} n(\zeta), R_{2}\right)
$$

and, in the case $R_{1}>0$,

$$
D\left(f(\zeta)-R_{1} n(\zeta), R_{1}\right) \leqslant f(U)
$$

where $D(a, R)$ is the open disc of radius $R$ cenetred at $a$.

For a function $f(z)$ in the class $C V\left(R_{1}, R_{2}\right)$, Goodman [2] obtained bounds for $d$ and $d^{*}$, where $d$ and $d^{*}$ are respectively the distances of the nearmost and the farthermost points on $\partial f(U)$ from the origin. Thus he proved that 


$$
R_{2}-\sqrt{R_{2}^{2}-R_{2}} \leq d \leq R_{1}-\sqrt{R_{1}^{2}-R_{1}}
$$

and

$$
R_{1} \leq \frac{d^{2}}{2 d-1} \leq R_{2}
$$

where the right hand side inequality in (4) and the left hand side

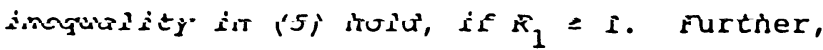

$$
d^{\star} \leq R_{2}+\sqrt{R_{2}^{2}-R_{2}} \text {. }
$$

Styer and Wright (7) observed that inequalities (4) and (6) continue to hold for the class $C V G\left(R_{1}, R_{2}\right)$. The method of proof of inequality (5) in [2] shows that this inequality also holds for the class CVG $\left(R_{1}, R_{2}\right)$ and is sharp. These inequalities are necessary conditions on $R_{1}$ and $R_{2}$ in terms of $d=d(f)$ for a function $f(z)$ to be in the class $C V G\left(R_{1}, R_{2}\right)$. However an analogue of these conditions in terms of $d^{*}$ is not known. Further, lower bound on $|f(z)|$, distortion properties involving $d^{*}$ or bound on $\left|\arg f^{\prime}(z)\right|$ for functions $f(z)$ in the class $C V G\left(R_{1}, R_{2}\right)$ have not been investigated so far.

section 2 is aimed at the determination of necessary and sufficient conditions for $R_{1}$ to be equal to $R_{2}$, if the function $f(z)$ is in the class $C V G\left(R_{1}, R_{2}\right)$. In this section analogues of conditions (4) and (5) involving $d^{\star}$ in place of $d$, for the functions in the class CVG $\left(R_{1}, R_{2}\right)$ are also found. Section 3 consists of theorems on the growth of $|f(z)|$ for functions $f(z)$ in the class $\operatorname{CVG}\left(R_{1}, R_{2}\right)$. Finally, Section 4 consists of a distortion theorem for the class $\operatorname{CVG}\left(R_{1}, R_{2}\right)$ and a rotation theorem for the class $\operatorname{CVG}\left(R_{1}, R_{2}\right)$.

2. PRELIMINARIES.

For a function $f \in \operatorname{CVG}\left(R_{1}, R_{2}\right)$, we first find some relations between the smallest and the largest distances of the image curve $\partial f(U)$ from the origin. We first prove the following lemma :

LEMMA 1. Let $f \in \operatorname{CVG}\left(R_{1}, R_{2}\right)$. If $R_{1}=R_{2}=R<\infty$, then

(i) $\quad d=\inf _{\zeta \in \partial(U)}|\zeta|=R-\sqrt{R^{2}-R}$.

(ii) $\quad f(U)=D\left(\left(\sqrt{R^{2}-R}\right) e^{i \alpha}, R\right)$, for some real $\alpha$.

(iii) $f(z)=e^{i \alpha} F_{R}\left(z e^{-i \alpha}\right)$, where $F_{R}(z)=\frac{z}{1-\sqrt{1-(1 / R)} z}, z \in U$

(iv) $\quad \mathrm{d}^{*}=\sup _{\zeta \in \partial f(U)}|\zeta|=R+\sqrt{\mathrm{R}^{2}-\mathrm{R}}$

PROOF.

(i) Follows by (4)

(ii) By the definition of CVG $\left(R_{1}, R_{2}\right)$, if $R_{1}=R_{2}=R<\infty, f(U)$ is a 
disc of radius $R$. If the center of the disc is at $\omega_{0}=r_{0} e^{i \alpha}$, $\alpha$ real, then

$$
r_{0}=R-d=\sqrt{R^{2}-R}
$$

or, equivalently,

$$
f(U)=D\left(\left(\sqrt{R^{2}-R}\right) e^{i \alpha}, R\right)
$$

(iii) $F_{R}(z)$ maps $U$ conformally onto the disc $D\left(\sqrt{R^{2}-R}, R\right)$. Thus,

$$
f(z)=e^{i \alpha} F_{R}\left(z e^{-i \alpha}\right) .
$$

(iv) Since $f(U)$ is a disc, $d+d^{*}=2 R$. Consequently, by (i),

$$
d^{\star}=R+\sqrt{R^{2}-R} \text {. }
$$

REMARK. The function $F_{R}(z)$ of Lemma 1 with $R=R_{2}$ (denoted as $F_{R_{2}}(z)$ in the sequel) was first used by Goodman [2] as an extremal function for a number of problems concerning $C V\left(R_{1}, R_{2}\right)$.

PROPOSITION 1. If $f \in \operatorname{CVG}\left(R_{1}, R_{2}\right)$, then

$$
1 \leq \frac{\left(d^{\star}\right)^{2}}{2 d^{\star}-1} \leq R_{2} \text {. }
$$

The inequalities are sharp for the function $F_{R_{2}}(z), R_{2} \geq 1$, of Lemma 1 ( $\mathrm{i} i \mathrm{i})$.

PROOF. Let $\psi(x)=x^{2} /(2 x-1)$. It is clear that $\psi(x)$ is increasing in $x$ if $1 \leq x<\infty$ and is decreasing in $x$ if $1 / 2 \leq x<1$. Thus inequality (7) follows from inequalities (6) and (5). If $\mathrm{d}^{\star}=\infty$, inequality (7) follows from Definition 2 .

The function $F_{R_{2}}(z)$ of Lemma 1 (iii) is in the class $\operatorname{CVG}\left(R_{1}, R_{2}\right)$ with $d^{\star}=1 /\left(1-\sqrt{1-1 / R_{2}}\right.$ and gives sharpness for inequality (7).

REMARK. For $f \in \operatorname{CVG}\left(R_{1}, R_{2}\right)$, inequality (7) sometimes gives a better lower bound on $R_{2}$ than that of inequality (5). In fact, $\left(d^{*}\right)^{2} /\left(2 d^{\star}-1\right)>d^{2} /(2 d-1)$, if and only if $d(2 d-1)<d^{*}$. There does exist a function in the class $\operatorname{CVG}\left(R_{1}, R_{2}\right)$ satisfying $d /(2 d-1)<d^{*}$; consider for example, $f(z)=2 \log (1-z / 2)^{-1} \in \overline{C V}(1,2 / \sqrt{3})$.

PROPOSITION 2. If $f \in \operatorname{CVG}\left(R_{1}, R_{2}\right)$ with $R_{1} \geq 1$, then

$$
\frac{\left(d^{\star}\right)^{2}}{2 d^{\star}-1} \geq R_{1} \text {. }
$$


and

$$
d^{*} \geq R_{1}-\sqrt{R_{1}^{2}-R_{1}}
$$

The inequalities are sharp when $R_{1}=R_{2}$.

PROOF. Let $d^{\star}<\infty$ and $f\left(e^{i \theta} 0, \in \partial f(U)\right.$ be such that $d^{\star}=\left|f\left(e^{i \theta} 0\right)\right|$, for some real $\theta_{0}$. By making a suitable rotation of $f(z)$, we may assume that $f\left(e^{i \theta} 0\right)=-d^{*}$. Then the unit exterior normal to $\partial f(U)$ at $f\left(e^{i \theta} 0\right)$ is $n\left(e^{i \theta} 0\right)=-1$. And, by the containment relation (3), we have

$$
D\left(R_{1}-d^{\star}, R_{1}\right) \leq f(U)
$$

equivalently,

$$
\frac{B z}{1-A z}<f(z)
$$

where $B=\left(2 R_{1}-d^{*}\right) d^{*} / R_{1}$ and $A=\left(R_{1}-d^{*}\right) d^{\star} / R_{1}$ for $R_{1}>0$. This implies $B \leq 1$, or,

$$
\frac{\left(d^{*}\right)^{2}}{2 d^{*}-1} \geq R_{1}
$$

which is inequality ( 8 ). The case $R_{1}=0$ is trivial. When $d^{*}=\infty$, inequality (8) follows directly.

Inequality (9) follows from inequality (8) and Definition 2. The sharpness of inequalities ( 8 ) and (9) follows by considering the function $F_{R_{2}}(z)$ of Lemma 1 (iii).

COROLLARY. If $f \in C V G\left(R_{1}, R_{2}\right)$, then

$$
R_{1} \leq \frac{\left(d^{\star}\right)^{2}}{2 d^{\star}-1} \leq R_{2}
$$

PROOF. Proposition 1 and inequality (8), together, give the corollary.

REMARKS. (i) For $f \in \operatorname{CVG}\left(R_{1}, R_{2}\right)$ with $R_{1} \geq 1$, it is easily seen that inequality (8) sometimes gives better upper bound for $R_{1}$ than that given by inequality (5). In fact, $\left(d^{*}\right)^{2} /\left(2 d^{*}-1\right)<d^{2} /(2 d-1)$, if and only if, $d^{*}<d /(2 d-1)$. There does exist a function in the class $\operatorname{CVG}\left(R_{1}, R_{2}\right)$, with $R_{1} z 1$, satisfying $d^{*}<d /(2 d-1)$; consider, for example, $f(z)=e^{z}-1 \in \overline{C V}(1, \infty)$.

(ii) For the function $f \in \overline{C V}\left(R_{1}, R_{2}\right)$ with $R_{1}<1$, inequality

(8) is not sharp, because $R_{1}<1 \leq\left(d^{\star}\right)^{2} /\left(2 d^{\star}-1\right)$.

3. GROWTH OF $|F(Z)|$.

For $f \in C V\left(R_{1}, R_{2}\right)$, Goodman $([2],[3])$ found that

$$
|f(z)| \leq 2 R_{2}-d
$$




$$
|f(z)| \leq \frac{r d\left(2 R_{2}-d\right)}{R_{2}(1-r)+r d}
$$

in the disc $|z|=r \leq 1$ where $d=\underset{\zeta \in \partial f(U)}{\inf }|\zeta|$. Both the inequalities are sharp. His proof shows that inequality (11) continues to hold for the class CVG $\left(R_{1}, R_{2}\right)$ also. However, analogues of inequalities (11) and (12), involving $\mathrm{d}^{*}=\sup _{\zeta \in \partial f(U)}|\zeta|$, are not known. In this section these analogues are derived.

Goodman [3] also showed that, if $f \in C V\left(R_{1}, R_{2}\right)$, then

$$
|f(z)| \leq r \frac{R_{2}}{R_{2}-r \sqrt{R_{2}^{2}-R_{2}}}
$$

for $|z|=r \in\left(r^{\star}, 1\right)$ where $r^{\star}=2 R_{2}\left(R_{2}-d\right) /\left(2 R_{2}\left(R_{2}-d\right)+d^{2}\right)$ and the inequality is sharp. In this section an analogous inequality for the functions in the class CVG $\left(R_{1}, R_{2}\right)$ is found wherein the number $r$ is independent of $d$.

In the following proposition, an analogue of inequality (11) involving $d^{*}$ in place of $d$ is found. In Theorem 1, an improvement of this proposition will be obtained.

PROPOSITION 4. If $f \in C V G\left(R_{1}, R_{2}\right)$ with $R_{2}<\infty$, then

$$
|f(z)| \leq r\left(R_{2}+\left|R_{2}-d^{*}\right|\right)
$$

in the disc $|z|=r \leq 1$. The inequality is sharp for $R_{1}=R_{2}$. PROOF. From the definition of $\mathrm{d}^{*}$, we have that

$$
|f(z)| \leq d^{*}
$$

in the disc $|z|=r \leq 1$. The triangle inequality and Schwarz lemma together with the above inequality completes the proof of (13).

For the function $F_{R_{2}}(z)$ of Lemma 1 (iii), $R_{1}=R_{2}$, and $\left|F_{R_{2}}(1)\right|=1 /\left(1-\sqrt{1-1 / R_{2}}\right)=R_{2}+\left|R_{2}-d^{*}\right|$. Thus, the sharpness of inequality (13) follows.

COROLLARY. If $f \in \operatorname{CVG}\left(R_{1}, R_{2}\right)$ wi.th $d^{*} \leq R_{2}$, then

$$
|f(z)| \leq r\left(2 R_{2}-d^{*}\right)
$$

in the $\operatorname{disc}|z|=r \leq 1$.

PROOF. The inequality in the corollary is straightforward in view of inequality (13).

REMARKS. (i) The corollary improves Goodman's result [2] given by inequality (11).

(ii) The functions $f(z)$ in the class $\operatorname{CVG}\left(R_{1}, R_{2}\right)$ satisfying $d<d^{*}<R_{2}<\infty$ do exist as can be seen from the following example. For integer $k^{2} z 2$ and $0<a<1 / k^{2}$, the binomial $p_{k}(z)=z+a z k \in \operatorname{CVG}\left(R_{1}, R_{2}\right)$ 
with $R_{2}=(1-k a)^{2} /\left(1-k^{2} a\right)$. Further, for $p_{k}(z), d=1-a<d^{\star}=1+a$, so that for $k=2, d^{\star}<R_{2}$ for $1 / 8<a<1 / 4$ and for $k \geq 3$, $\mathrm{d}^{\star}<\mathrm{R}_{2}$ for $0<\mathrm{a}<1 / \mathrm{k}^{2}$.

(iii) An analogue of inequality (13) involving $R_{1}$ can also be found. Thus, if $f \in \operatorname{CVG}\left(R_{1}, R_{2}\right)$ with $R_{2}<\infty$, then

$$
|f(z)| \leq r\left(R_{1}+\left|R_{1}-d^{\star}\right|\right)=r d^{\star} \leq r\left(R_{2}+\left|R_{2}-d^{\star}\right|\right)
$$

in the disc $|z|=r \leq 1$. The above inequality is sharp for $R_{1}=R_{2}$. Next, a growth theorem is derived for the class CVG $\left(R_{1}, R_{2}\right)$ with the help of the following lemma:

LEMMA 2 [5]. If $F(z)$ is in $C V$ and $f(z)$ is convex and univalent in $U$, then $f(z) \curlywedge F(z)$ in $U$ implies that

$$
|f(z)| \leq|F(z)|
$$

in the $\operatorname{disc}|z|<\underline{R}$, where $\underline{R} \cong 0.543$ is the least positive root of

$$
\operatorname{arc} \sin x+2 \text { arc } \tan x=\frac{\pi}{2} \text {. }
$$

THEOREM 1. If $f \in \operatorname{CVG}\left(R_{1}, R_{2}\right)$ with $0<R_{1} \leq R_{2}<\infty$, then

$$
\frac{r d^{\star}\left|2 R_{1}-d^{\star}\right|}{R_{1}(1-r)+r d^{\star}} \leq|f(z)| \leq \frac{r d^{\star}\left|2 R_{2}-d^{\star}\right|}{R_{2}-\left|R_{2}-d^{\star}\right| r}
$$

where $|z|=r$, the left hand side inequality holds in the disc $|z|<\underline{R}, \underline{R}$ is as in Lemma 2 and the right hand side inequality holds in the disc $|z| \leq 1$. Both the inequalities are sharp.

PROOF. By making a suitable rotation of $f(z)$ we may obtain that $f\left(e^{i \theta} 0\right)=-d^{\star}=-\sup _{\zeta \in \partial f(U)}|\zeta|$, for some $\theta_{0}$ real. We have $n\left(e^{i \theta} 0\right)=-1$.

Now, the by containment relation (2), we get

or,

$$
f(U) \subseteq D\left(R_{2}-d^{\star}, R_{2}\right)
$$

$$
f(z)<\frac{B z}{1-A z}
$$

where $B=d^{\star}\left(2 R_{2}-d^{\star}\right) / R_{2}$ and $A=\left(R_{2}-d^{*}\right) / R_{2}$.

The inverse of the function $g(z)=B z /(1-A z)$ is $h(z)=z /(A z+B)$ and the function $\eta(z)=$ (hof)(z) satisfies the conditions of Schwarz lemma. So,

$$
|f(z)| \leq r(|A f(z)|+B)
$$

in the disc $|z|=r \leq 1$. This implies that

$$
|f(z)| \leq \frac{r B}{1-r|A|} \text {. }
$$

By substituting the values of $A$ and $B$ in this, the right hand side inequality of (14) is obtained. 
Now, to prove the left hand side inequality in (14), we apply the containment relation (3) and obtain

$$
\frac{B^{\star} Z}{1-A^{\star} z}<f(z)
$$

where $B^{\star}=d^{\star}\left(2 R_{1}-d^{\star}\right) / R_{1}$ and $A^{\star}=\left(R_{1}-d^{\star}\right) / R_{1}$.

Further,

$$
\begin{aligned}
\left|\frac{B^{*} z}{1-A^{\star} z}\right| & \geq \frac{\left|B^{*}\right| r}{1+\left|A^{\star}\right| r} \\
& =\frac{r d^{\star}\left|2 R_{1}-d^{\star}\right|}{R_{1}+\left(d^{\star}-R_{1}\right) r}
\end{aligned}
$$

in the disc $|z|=r<1$.

Hence, by Lemma 2 , we have that

$$
\frac{r d^{\star}\left|2 R_{1}-d^{\star}\right|}{R_{1}(1-r)+r d^{\star}} \leq\left|\frac{B^{\star} z}{1-A^{\star} z}\right| \leq|f(z)|
$$

in the disc $|z|<B$ where $\underline{R}$ is as in Lemma 2. This gives the left hand side inequality of (14).

The function $F_{R_{2}}(z)$ of Lemma l(iii), is in the class $\operatorname{CVG}\left(R_{2}, R_{2}\right)$. For this function, $d^{*}=1 /(1-a) \geq R_{2}$ so that $r d^{\star}\left(2 R_{2}-d^{*}\right) /\left(R_{2}-\left|R_{2}-d^{*}\right| r\right)=r /(1-a r)$ and $r d^{*}\left|2 R_{1}-d^{*}\right| /\left(R_{1}(1-r)+r d^{*}\right)$ $=r /(1+a r)=\left|F_{R_{2}}(-r)\right|$ where $a=\sqrt{1-1 / R_{2}}$ and now equality is attained in inequality (14).

REMARKS. (i) For $f \in C V G\left(R_{1}, R_{2}\right)$ with $R_{2}<\infty$ and $r=1$ the upper bound of $|f(z)|$ in inequality (14) is larger than that given by inequality (13). For the function $F_{R_{2}}(z)$ of Lemma 1 (iii), both the bounds are equal. For $r<1$, the upper bound given by inequality (14) is better than that given by inequality (13).

(ii) From the proof of Theorem 1,it can be observed that inequality (14) with $\mathrm{d}^{*}$ replaced by d everywhere, continues to remain true and sharp; i.e., if $f \in \operatorname{CVG}\left(R_{1}, R_{2}\right)$ with $0 \leq R_{1} \leq R_{2}<\infty$, then

$$
\frac{r d\left|2 R_{1}-d\right|}{R_{1}+\left|R_{1}-d\right| r} \leq|f(z)| \leq \frac{r d\left|2 R_{2}-d\right|}{R_{2}(1-r)+r d}
$$

where $|z|=r$, the left-hand side inequality holds in the disc $|z|<\underline{R}, \underline{R}$ is as in Lemma 1 , and the right hand side inequality holds in the disc $|z| \leq 1$. The same function $F_{R_{2}}(z)$ of Lemma 1 (iii) gives the sharpness in this inequality also.

(iii) Let $Q\left(r, R_{2}, x\right)=x\left(2 R_{2}-x\right) /\left(R_{2}-\left|R_{2}-x\right| r\right)$.

It can be seen that for $r \in\left[r^{*}, 1\right)$, the function $Q\left(r, R_{2}, x\right)$ is decreasing in $x$ for $x \leq R_{2}$ and hence the upper bound of $|f(z)|$ in 
inequality (14) is better than that in inequality

(16) for $R_{2} \geq d^{*}$ where $r^{\star}=2 \sqrt{R_{2}^{2}-R_{2}} /\left(2 R_{2}-1\right)$.

(iv) Let $P\left(r, R_{1}, x\right)=x\left|2 R_{1}-x\right| /\left(R_{1}+\left|R_{1}-x\right| r\right)$. It can be seen that for $r \in(0, \underline{R}), \underline{R}$ is as in Lemma 2 , the function $P\left(r, R_{1}, x\right)$ is decreasing in $x$ for $x \in\left[R_{1}, 2 R_{1}\right]$ and hence the lower bound of $|f(z)|$ in inequality (16) is better than that in inequality (14) for $R_{1} \leq d \leq d^{*} \leq 2 R_{1}$; the last inequality does hold for the function $p_{3}(z)=z+a z^{3} \in \operatorname{CVG}\left((1+3 a)^{2} /(1+9 a), R_{2}\right)$, where $0 \leq a \leq 1 / 15$.

(v) For $f \in \overline{C V}\left(R_{1}, R_{2}\right)$ with $R_{1}<R_{2}$, strict inequality holds in the right hand side of the inequality (14), because, when equality holds, inequality (15) gives that $f(z)=c z /(1-D z)$ where $C=e^{i \phi} d^{\star}\left(2 R_{2}-d^{\star}\right) / R_{2}$ and $D=e^{i \phi} d^{*}\left(R_{2}-d^{\star}\right) / R_{2}$, $\phi$ real, so that $f(z)$ has $R_{1}=R_{2}$.

For $f \in C V G\left(R_{1}, R_{2}\right)$, the upper bound of $|f(z)|$ in inequality (14) (or (16)) is dependent on $d^{\star}$ (or $d$ ). The following theorem gives an upper bound of $|f(z)|$ that is independent of both $d$ and $d^{*}$.

THEOREM 2. If $f \in \operatorname{CVG}\left(R_{1}, R_{2}\right)$ with $R_{2}<\infty$, then

$$
|f(z)| \leq r \frac{R_{2}}{R_{2}-\sqrt{R_{2}^{2}-R_{2}}}
$$

where $|z|=r \in\left[r^{\star}, 1\right]$ and $r^{\star}=2 \sqrt{R_{2}^{2}-R_{2}} /\left(2 R_{2}-1\right)$. The inequality is sharp.

PROOF. Set $Q\left(r, R_{2}, d\right)=d\left(2 R_{2}-d\right) /\left(R_{2}(1-r)+r d\right)$. Then, $r Q\left(r, R_{2}, d\right)$ is the upper bound of $|f(z)|$ in inequality (16). Let $r^{\star}=2 \sqrt{R_{2}^{2}-R_{2}} /\left(2 R_{2}-1\right)$. For $r \in\left[r^{\star}, 1\right]$, the function $r Q\left(r, R_{2}, d\right)$ is decreasing in d. By inequality (4), we have $d \geq R_{2}-\sqrt{R_{2}^{2}-R_{2}}$. Hence, for $r \in\left[r^{\star}, 1\right]$, we may replace $d$ by $R_{2}-\sqrt{R_{2}^{2}-R_{2}}$ in $r Q\left(r, R_{2}, d\right)$ and obtain the assertion from inequality (16).

The function $F_{R_{2}}(z)$ of Lemma 1(iii), gives sharpness in inequality (17) for $z=r$.

REMARKS. (i) For $f \in \operatorname{CVG}\left(R_{2}, R_{2}\right)$, the upper bound of $|f(z)|$ in inequality (17) is better than that in inequality (13). Indeed, for the function $Q^{\star}\left(r, R_{2}\right)=r R_{2} /\left(R_{2}-r \sqrt{R_{2}^{2}-R_{2}}\right)$ we have

$Q^{\star}\left(r, R_{2}\right) \leq r R_{2} /\left(R_{2}-\sqrt{R_{2}^{2}-R_{2}}\right)=r\left(R_{2}+\sqrt{R_{2}^{2}-R_{2}}\right)$ for $r \in\left[2 \sqrt{R_{2}^{2}-R_{2}} /\left(2 R_{2}-1\right), 1\right] \cdot$

(ii) If $f \in \overline{\mathrm{CV}}\left(\mathrm{R}_{1}, \mathrm{R}_{2}\right)$ and equality holds in (17), then as in Remark (v) following the proof of Theorem 1 , we obtain that $R_{1}=R_{2}$. Hence strict inequality holds in (17) when $R_{1}<R_{2}$.

In the following result an upper bound on $|f(z)|$ involving both $R_{1}$ and $R_{2}$ is obtained. 
THEOREM 3. If $f \in \operatorname{CVG}\left(R_{1}, R_{2}\right)$ with $1 \leq R_{1} \leq R_{2}<\infty$, then

$$
|f(z)| \leq \frac{r \alpha_{1}\left(2 R_{2}-\alpha_{1}\right)}{R_{2}(1-r)+r \alpha_{1}}
$$

in the disc $|z|=r \leq r^{\star *}=2 R_{2}\left(R_{2}-\alpha_{1}\right) /\left(2 R_{2}\left(R_{2}-\alpha_{1}\right)+\alpha_{1}^{2}\right)$ and $\alpha_{1}=R_{1}-\sqrt{R_{1}^{2}-R_{1}}$. The inequality is sharp for $R_{1}=R_{2}$.

PROOF. Set $Q\left(r, R_{2}, d\right)=d\left(2 R_{2}-d\right) /\left(R_{2}(1-r)+r d\right)$. Then, $r Q\left(r, R_{2}, d\right)$ is the upper bound of $|f(z)|$ in inequality (16). Let $r^{\star \star}=2 R_{2}\left(R_{2}-\alpha_{1}\right) /\left(2 R_{2}\left(R_{2}-\alpha_{1}\right)+\alpha_{1}^{2}\right)$ where $\alpha_{1}=R_{1}-\sqrt{R_{1}^{2}-R_{1}}$. For $r \in\left[0, r^{\star \star}\right]$, the function $r Q\left(r, R_{2}, d\right)$ is increasing in $d$. By inequality (4), we have that $d \leq R_{1}-\sqrt{R_{1}^{2}-R_{1}}$. Thus, we may replace $d$ by $R_{1}-\sqrt{R_{1}^{2}-R_{1}}$ in $r Q\left(r, R_{2}, d\right)$ and obtain the assertion from inequality (16).

For $R_{1}=R_{2}$, the upper bound $r Q\left(r, R_{2}, \alpha_{1}\right)$ equals $r R_{2} /\left(R_{2}-r \sqrt{\left.R_{2}^{2}-R_{2}\right)}\right.$. The function $F_{R_{2}}(z)$ of Lemma $l(i i i)$ gives sharpness in inequality (18) for $z=r$.

REMARKS. (i) The number $r^{\star}$ defined in Theorem 2 is larger than $r^{\star \star}$ defined in Theorem 3 . Both are equal, if and only if, $R_{1}=R_{2}$.

(ii) For $f \in \overline{C V}\left(R_{1}, R_{2}\right)$ with $1 \leq R_{1}<R_{2}<\infty$, strict inequality holds in (18); for, when equality holds, it can be seen as in Remark (v) following the proof of Theorem 1 , that $R_{1}=R_{2}$, a contradiction.

4. DISTORTION AND ROTATION THEOREMS.

For $f \in C V\left(R_{1}, R_{2}\right)$, Goodman [3] found that

$$
\left|f^{\prime}(z)\right| \leq \frac{R_{2}}{1-r^{2}}
$$

in the disc $|z|=r<1$. The function $F_{R_{2}}(z)$, of Lemma $1(i i i)$, for $R_{2}=1 /\left(1-r^{2}\right)$ shows that inequality (19) is sharp for each $r \in(0,1)$. From the proof of inequality (19), we observe that inequality (19) continues to hold for the class $\operatorname{CVG}\left(R_{1}, R_{2}\right)$. However, an analogue of inequality (19) in terms of $\mathrm{d}^{*}=\sup _{\zeta \in \partial f(U)}|\zeta|$ is not known. In this section a result in this direction is found for the class $\operatorname{CVG}\left(R_{1}, R_{2}\right)$.

Finally, in this section, a rotation theorem is derived for the class $C V\left(R_{1}, R_{2}\right)$. Its validity for the class $C V G\left(R_{1}, R_{2}\right)$ remains open for investigation.

The following lemma is needed in the sequel:

LEMMA 3 [7]. If $f \in \underline{S}$ with $g(z)<f(z)$ in $U$ and $g^{\prime}(0) z 0$, then $\left|g^{\prime}(z) \leq\right| f^{\prime}(z) \mid$ in the disc $|z| \leq 3-\sqrt{8} \cong 0.171$. 
THEOREM 4. If $f \in \operatorname{CVG}\left(R_{1}, R_{2}\right)$ with $0<R_{1} \leq R_{2}<\infty$, then

$$
\frac{R_{1} d^{*}\left|2 R_{1}-d\right|}{\left(R_{1}(1-r)+r d^{\star}\right)^{2}} \leq\left|f^{\prime}(z)\right| \leq \frac{R_{2} d^{\star}\left(2 R_{2}-d^{*}\right)}{\left(R_{2}-\left|R_{2}-d^{\star}\right| r\right)^{2}}
$$

in the disc $|z|=r \leq 3-\sqrt{8}$. The inequalities are sharp for $R_{1}=R_{2}$.

PROOF. As in the proof of Theorem 1, we obtain

$$
f(z)<\frac{B z}{1-\bar{A} z}
$$

where $B=d^{\star}\left(2 R_{2}-d^{\star}\right) / R_{2}$ and $A=\left(R_{2}-d^{\star}\right) / R_{2}$. This and Lemma 3 together give

$$
\left|f^{\prime}(z)\right| \leq \frac{B}{(1-|A| r)^{2}}
$$

in the disc $|z|=r \leq 3-\sqrt{8}$. By substituting the values of $A$ and $B$ in this inequality, the right hand side inequality of $(20)$ is obtained.

To prove the left-hand side of the inequality (20), we have, as in the proof of Theorem 1,

$$
\frac{B^{\star} z}{1-A^{\star} z}<f(z)
$$

where $B^{\star}=d^{\star}\left(2 R_{1}-d^{\star}\right) / R_{1}$ and $A=\left(R_{1}-d^{\star}\right) / R_{1}$. Therefore, by Lemma 3 ,

$$
\begin{aligned}
\left|f^{\prime}(z)\right| & \geq\left|\frac{B^{\star}}{\left(1-A^{\star} z\right)^{2}}\right| \\
& \geq \frac{R_{1} d^{\star}\left|2 R_{1}-d^{\star}\right|}{\left(R_{1}(1-r)+r d^{\star}\right)^{2}}
\end{aligned}
$$

in the disc $|z|=r \leq 3-\sqrt{ } 8$, which is the left-hand side of the inequality (20).

For the function $F_{R_{2}}(z)$ of Lemma $1(i i i), R_{1}=R_{2}$ and $d^{\star}=1 /(1-a)$ so that $R_{2} d^{\star}\left(2 R_{2}-d^{*}\right) /\left(R_{2}-\left|R_{2}-d^{*}\right| r\right)^{2}=1 /(1-a r)^{2}=\left|F_{R_{2}}^{\prime}(r)\right|$ and $R_{1} d^{\star}\left(2 R_{1}-d^{\star}\right)\left|/\left(R_{1}(1-r)+r d^{\star}\right)^{2}=1 /(1+a r)^{2}=\right| F_{R_{2}}^{\prime}(-r) \mid$ where $a=\sqrt{1-1 / R_{2}}$ so that equality is attained in inequality (20).

REMAKRS. (i) For $f \in C V G\left(R_{1}, R_{2}\right)$, the upper bound of $\left|f^{\prime}(z)\right|$ in inequality $(20)$ is better than that in inequality (19). The sharp function given in the proof of Theorem 4 is independent of the point under consideration whereas the sharp function used for inequality (19) is dependent on the point.

(ii) From the proof of Theorem 4, it can be seen that inequality $(20)$ continues to remain true with $\mathrm{d}^{*}$ replaced by $d$ everywhere, i.e., for $f \in C V G\left(R_{1}, R_{2}\right)$ with $0 \leq R_{1} \leq R_{2}<\infty$, we have 
that

$$
\frac{R_{1} d\left|2 R_{1}-d\right|}{\left(R_{1}+\left|R_{1}-d\right| r\right)^{2}} \leq\left|f^{\prime}(z)\right| \leq \frac{R_{2} d\left|2 R_{2}-d\right|}{\left(R_{2}(1-r)+r d\right)^{2}}
$$

in the disc $|z|=r \leq 3-\sqrt{8}$.

(iii) For $f \in C V G(R, R)$ with $d^{\star} \leq R \leq 1 /(12 \sqrt{2}-16)$ and $\sqrt{R^{2}-R} / R \leq r \leq 3-\sqrt{8}$, the upper bound of $\left|f^{\prime}(z)\right|$ in inequality (20) is better than that of the inequality (21).

(iv) For $f \in C V G\left(R_{1}, R_{2}\right)$ with $R_{2}<\infty$, the lower bounds of $\left|f^{\prime}(z)\right|$ in inequalities $(20)$ and (21) are equal by proposition 3 . similarly, the upper bounds of $\left|f^{\prime}(z)\right|$ are also equal.

(v) For $f \in \operatorname{CVG}\left(R_{1}, R_{2}\right)$ with $R_{1} \leq d \leq d^{*} \leq 2 R_{1}$, the lower bound of $\left|f^{\prime}(z)\right|$ in inequality $(21)$ is better than that in inequality (20).

Finally, we prove a rotation theorem for the class $C V\left(R_{1}, R_{2}\right)$. Its validity for the class $f \in C V G\left(R_{1}, R_{2}\right)$ remains open for investigation.

THEOREM 5. If $f \in C V\left(R_{1}, R_{2}\right)$ with $R_{2}<\infty$, then $\left|\arg f^{\prime}(z)\right| \leq 2 \ln \frac{\sqrt{R_{2}(1+r)}+c\left(r, R_{2}\right)}{\sqrt{1-r}\left(\sqrt{R_{2}}+\sqrt{\left.R_{2}-1\right)}\right.}+\frac{2}{\sqrt{R_{2}}}\left(\sqrt{R_{2}-1}-\frac{c\left(r, R_{2}\right)}{\sqrt{1+r}}\right)$ in the disc $|z|=r<1$ where $C(r, R)=\sqrt{R(1+r)-(1-r)}$.

PROOF. For each fixed $\lambda$ in $U$, the function

$$
\begin{aligned}
& g(z) \equiv \frac{f\left(\frac{z+\lambda}{1+\bar{\lambda} z}\right)-f(\lambda)}{f^{\prime}(\lambda)\left(1-|\lambda|^{2}\right)} \\
& =z+c_{2}(\lambda) z^{2}+\ldots
\end{aligned}
$$

is $\operatorname{CV}\left(R_{1} / A(\lambda), R_{2} / A(\lambda)\right)$ where $A(\lambda)=\left|f^{\prime}(\lambda)\right|\left(1-|\lambda|^{2}\right)$. It is known [8] that if $g \in C V\left(R_{1}^{\prime}, R_{2}^{\prime}\right)$ then $\mid g^{\prime \prime}(0) / 2 ! \leq \sqrt{1-1 / R_{2}^{\prime}}$. Therefore,

$$
\left|c_{2}(\lambda)\right|=\left|\frac{f^{\prime \prime}(\lambda)\left(1-|\lambda|^{2}\right)}{2 f^{\prime}(\lambda)}-\bar{\lambda}\right| \leq\left(1-\frac{\left|f^{\prime}(\lambda)\right|\left(1-|\lambda|^{2}\right)}{R_{2}}\right)^{1 / 2}
$$

which, by using the distortion property $\left|f^{\prime}(\lambda)\right| \geq \frac{1}{(1+|\lambda|)^{2}}$ for the function $f(z)$ in $c v$, gives

$$
\left|\frac{f^{\prime \prime}(\lambda)\left(1-|\lambda|^{2}\right)}{2 f^{\prime}(\lambda)}-\bar{\lambda}\right| \leq \sqrt{1-\frac{1-|\lambda|}{R_{2}(1+|\lambda|)}} .
$$


Multiplying the above inequality by $2|\lambda| /\left(1-|\lambda|^{2}\right.$, we obtain

$$
\left|\frac{\lambda f((\lambda)}{f^{\prime}(\lambda)}-\frac{2|\lambda|^{2}}{1-|\lambda|^{2}}\right| \leq \frac{2|\lambda|}{1-|\lambda|^{2}} \sqrt{1-\frac{1-|\lambda|}{R_{2}(1+|\lambda|)}} \text {. }
$$

Replacing $|\lambda|$ by $\rho$ in the above inequality, we get

$$
-\frac{2 \rho}{1-\rho^{2}} \sqrt{1-\frac{1-p}{R_{2}(1+\rho)}} \leq \operatorname{Im}\left(\frac{\lambda f^{\prime \prime}(\lambda)}{f^{\prime}(\lambda)}-\frac{2 \rho}{1-\rho^{2}}\right) \leq \frac{2 \rho}{1-\rho^{2}} \sqrt{1-\frac{1-p}{R_{2}(1+\rho)}} .
$$

Thus,

$$
-\frac{2}{1-\rho^{2}} \sqrt{1-\frac{1-\rho}{R_{2}(1+\rho)}} \leq \frac{\partial \arg f^{\prime}(\lambda)}{\partial \rho} \leq \frac{2}{1-\rho^{2}} \sqrt{1-\frac{1-\rho}{R_{2}(1+\rho)}}
$$

since,

$$
\operatorname{Im}\left(\lambda \frac{f^{\prime \prime}(\lambda)}{f^{\prime}(\lambda)}-\frac{2 \rho^{2}}{1-\rho^{2}}\right)=\rho \frac{\partial}{\partial \rho} \arg f^{\prime}(\lambda) \text {. }
$$

Now, integrating the terms in inequality (22) along the straight linepath from $\lambda=0$ to $\lambda=\mathrm{re}^{i \theta}$, the required inequality follows.

\section{REFERENCES}

1. BIERNACKI, M. Sur les fonctions univalentes, Mathematica 12 $(1936), 49-64$.

2. GOODMAN, A.W. Convex functions of bounded type, Proc. Amer. Math. Soc. 92, (1984), 541-546.

3. More on convex functions of bounded type, Proc. Amer. Math. Soc. 97 (1986), 303-306.

4. MEJIA, D. and MINDA, D. Hyperbolic geometry in $k$-convex regions, Pacific J. Math. 141 (1990), 333-354.

5. TAO-SHING, SHAH. On the radius of superiority in subordination, Science Record 1 (1957), 53-57.

6. STUDY, E. Konforme Abbildung Einfachzusammenhangender Bereiche, Teubner, Leipzig and Berlin, 1913.

7. STYER, D. and WRIGHT, D.J. Convex functions with restricted curvature, Proc. Amer. Math. Soc. 109,(1990), 981-990.

8. WIRTHS, K.J. Coefficient bounds for convex functions of bounded type, Proc. Amer. Math. Soc. 103 (1988), 525-530. 


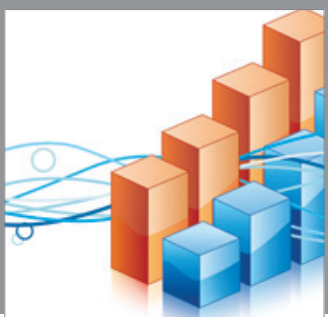

Advances in

Operations Research

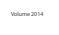

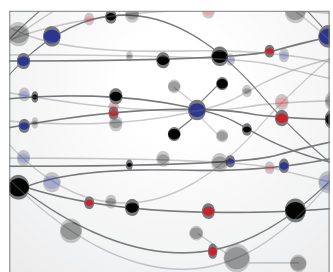

\section{The Scientific} World Journal
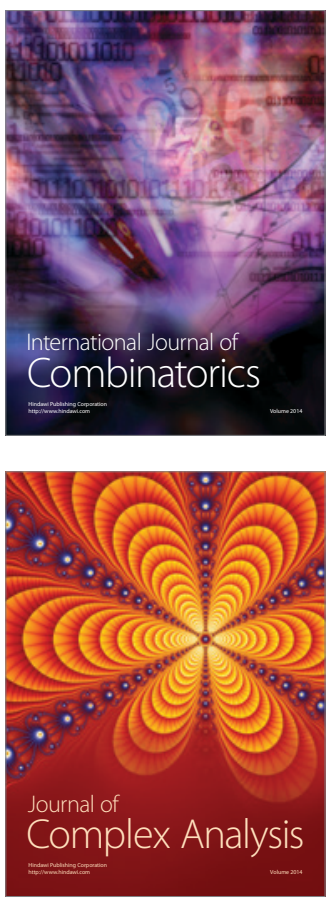

International Journal of

Mathematics and

Mathematical

Sciences
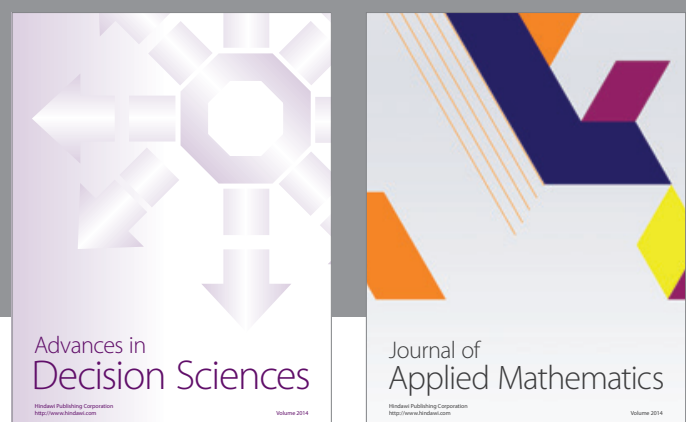

Journal of

Applied Mathematics
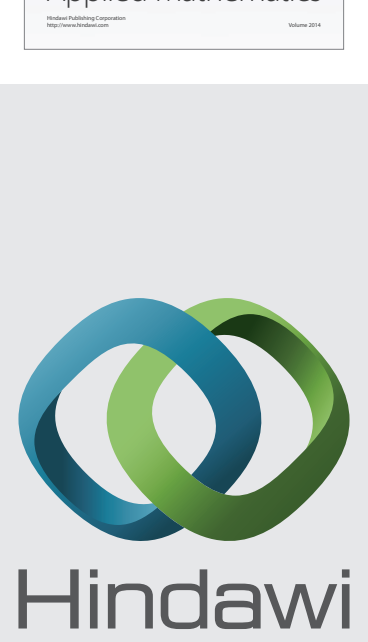

Submit your manuscripts at http://www.hindawi.com
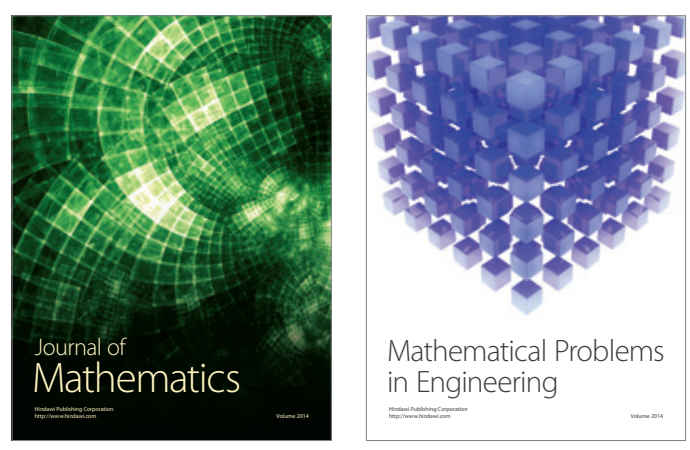

Mathematical Problems in Engineering
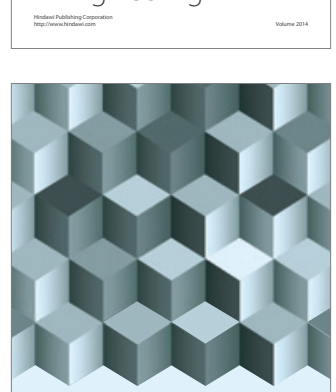

Journal of

Function Spaces
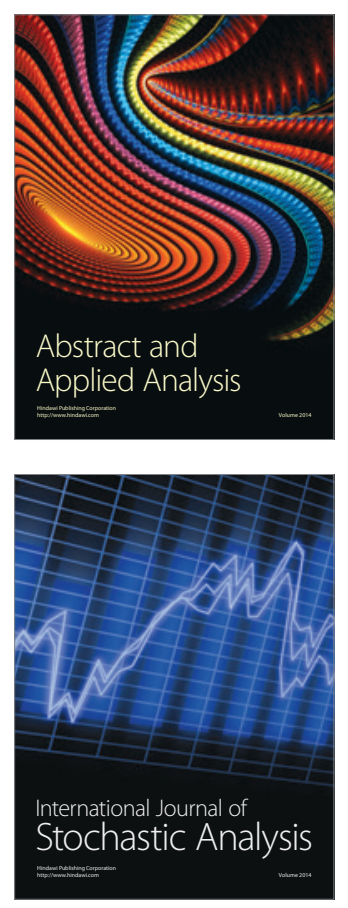

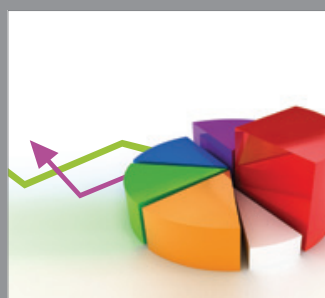

ournal of

Probability and Statistics

Promensencen
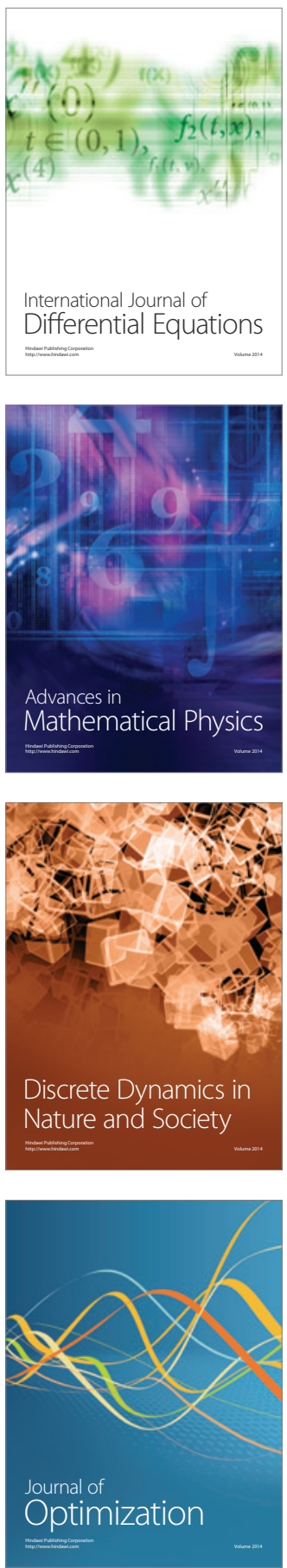